\title{
O consumo de alimentos orgânicos na cidade de Manaus (AM): o comércio de produtos e a sustentabilidade do setor
}

\section{Consumption of organic food in the city of Manaus (AM): the trade of products and the sector's sustainability}

\author{
Paula de Fátima da Cruz Lima, Aline Maria Meiguins de Lima², Sinaida Maria Vasconcelos de Castro ${ }^{2}$, Maria de Valdivia \\ Costa Norat Gomes ${ }^{4}$
}

\begin{abstract}
Resumo: A produção de alimentos orgânicos proporciona a oferta de produtos saudáveis; o uso sustentável da água e do solo; a integridade cultural das comunidades rurais; a sustentabilidade econômica e ecológica; e a eliminação do uso de organismos geneticamente modificados em qualquer fase do processo de produção, processamento, armazenamento, distribuição e comercialização. Considerando a importância ecológica da região Amazônica, foi avaliado o mercado de consumo de produtos alimentícios orgânicos em Manaus-Amazonas e caracterizado seu perfil de consumo, potencialidade e limitações. A pesquisa foi realizada junto a Associação de Produtores Orgânicos do Amazonas. Os resultados indicaram os consumidores são em sua maioria do sexo feminino, com faixa etária entre 30 a 40 anos, nível de escolaridade superior completo, em sua maioria, com uma renda superior a 4 salários mínimos. São clientes e consumidores fiéis, porém não avaliam com critério o alimento que estão adquirindo. Os produtores encontram dificuldades principalmente no apoio do poder público à produção e comercialização, com isso a oferta não é ampla e variada, havendo a competição com os preços dos produtos gerados sem a qualidade de orgânico.
\end{abstract}

Palavras-chaves: Alimentos orgânicos. Produção. Consumidores. Amazônia.

\begin{abstract}
The production of organic food provides the availability of healthy products; sustainable use of water and soil; cultural integrity of rural communities; the economic and ecological sustainability; and eliminating the use of genetically modified at any stage of the production process, processing, storage, distribution and sale. Considering the ecological importance of the Amazonia region, was evaluated the market consumption of organic food products in Manaus-Amazonas and characterized their consumption profile, potential and limitations. The research was conducted with the Organic Producers Association of Amazonas. The results indicated consumers are mostly female, aged between 30 to 40 years, level complete higher education, mostly with income exceeding 4 minimum wages. The customers are loyal, but do not evaluate with criteria the food that they are buying. The producers have difficulties with the government support to the production and marketing. Therefore, the offer is not wide and varied, and still there is the competition with the prices of products produced without the organic quality.
\end{abstract}

Key words: Organic foods. Production. Consumer. Amazonia.

\footnotetext{
*Autor para correspondência

Recebido para publicação em 02/01/2015; aprovado em 25/01/2015

${ }^{1}$ MSc. Programa de Pós-Graduação em Processos Construtivos e Saneamento Urbano, Universidade Federal do Pará. Manaus-AM,

paulafclima@yahoo.com.br

2 *Dra , Programa de Pós-Graduação em Processos Construtivos e Saneamento Urbano, Universidade Federal do Pará. Belém-Pa, ameiguins@ ufpa.br.

${ }^{2} \mathrm{Dr}^{\mathrm{a}}$. Universidade do Estado do Pará, Centro de Ciências e Planetário do Pará. Belém-Pa, sinaida@ cesupa.br.

${ }^{4}$ MSc. Programa de Pós-Graduação em Processos Construtivos e Saneamento Urbano, Universidade Federal do Pará. Belém-Pa, vnorat@ufpa.br.
} 


\section{INTRODUÇÃO}

As modificações que ocorreram no setor agroalimentar, ocasionadas pelo uso indiscriminado de agrotóxicos, fertilizantes e sementes modificadas geneticamente levaram os consumidores de alimentos orgânicos, a estabelecer uma relação entre saúde, alimentação e preocupação ambiental (HOEFKENS et al., 2011). Essa preocupação induziu a sociedade a entender que o alimento produzido de forma orgânica se torna um meio de prevenção do meio ambiente e manutenção da saúde (ARCHANJO et al., 2001).

A Lei Federal no 10. 831 de 23 de dezembro de 2003 define sistema de produção orgânico como todo sistema de produção cujas técnicas aperfeiçoam o uso dos recursos naturais e socioeconômicos disponíveis, respeitando a integridade cultural das comunidades rurais com o objetivo de obter a sustentabilidade econômica e ecológica, eli- pu colocar tudo no pluralminando o uso de organismos geneticamente modificados, em qualquer fase do processo de produção, processamento, armazenamento, distribuição e comercialização.

Borguini e Torres (2006) definem alimento orgânico como um alimento que foi produzido seguindo normas específicas, as quais não permitem o uso de qualquer agroquímico e que o alimento estejam certificados por empresa autorizada.

Segundo Ferraz et al. (2013) o cultivo de alimentos orgânicos foi identificado em mais de 120 países, onde existem mais de 600 mil agricultores orgânicos; dentre os continentes destacam-se em ordem crescente a Oceania (com a maior área agrícola cultivada organicamente), a Europa e a América Latina.

A produção de orgânicos, no Brasil, surgiu na década de 90, inicialmente na região Sul do País e veio crescendo $30 \%$ ao ano. Ao se disseminar pelas demais regiões, deixou de ser apenas uma simples produção de hortaliças sem venenos, transformando o País no segundo maior produtor de orgânicos do mundo, com $75 \%$ de sua produção exportada para a Europa, Estados Unidos e Japão (ALBUQUERQUE, 2011).

Os principais alimentos orgânicos produzidos no País são a soja (31\%), as hortaliças $(27 \%)$, o café $(25 \%)$, as frutas $(26 \%)$ e o palmito (18\%). Já os grandes produtores empresariais que exportam esse tipo de alimento representam $10 \%$ da produção (TERRAZZA; VALARIN, 2009). Destacam-se na produção para exportação a soja, o café, o cacau, o açúcar mascavo, a erva-mate, o suco de laranja, o mel, as frutas secas, a castanha de caju, os óleos essenciais, o óleo de palma, as frutas tropicais, o palmito, o guaraná e o arroz (BOURGUINI; TORRES, 2006).

Segundo Cremonezi et al. (2011), o IBD Certificações - Inspeções e Certificações Agropecuárias e Alimentícias apresentou, em 2011, um registro de 354 produtores de alimentos orgânicos certificados pela instituição no Brasil e o percentual de produtores por Estado, totalizando, ainda, segundo dados do IBD, uma produção de 1.565 produtos in natura e industrializados.

De acordo com Santos e Monteiro (2004), para que um produtor seja qualificado como orgânico é necessário se submeter a um processo rigoroso de investigação da sua produção, a fim de que a certificadora the atribua o selo de produto orgânico. No Brasil, existem 15 empresas certificadoras credenciadas ao Colegiado Nacional para a
Produção Orgânica (CNPOrg). Esse colegiado está vinculado ao Ministério da Agricultura, Pecuária e Abastecimento (MAPA) e tem como objetivo assessorar e acompanhar a implantação das normas de produção de alimentos orgânicos, avaliando e emitindo os pareceres conclusivos sobre os processos de credenciamento das entidades certificadoras.

$\mathrm{O}$ arcabouço legal para implantação do mercado de produtos orgânicos é necessário, porém não pode ser uma ação isolada, Lacaze (2009) argumenta que a fixação da cultura do consumo de alimentos orgânicos depende da avaliação do valor do produto e a disposição do consumidor em pagá-lo (NESS, et al., 2010; DIAZ, et al., 2011); além da percepção dos ganhos em termos de saúde pública e benefício ambiental que eles podem implicar ao usuário e ao produtor.

Roitner-Schobesberger et al. (2008) e Lairon (2010) discutem que os consumidores de produtos orgânicos tendem a ponderar no momento da compra sobre valor nutricional destes e a segurança alimentar (YIRIDOE et al., 2005). Autores como Bourn e Prescott (2002) e Lima Vianelo (2011), abordam a questão do aspecto "mais saudável" atribuído aos alimentos orgânicos, principalmente pela provável ausência de agrotóxicos e a maior concentração de nutrientes; no entanto, também levantam questões sobre a necessidade de estudos individuais dos alimentos e das formas de cultivo e controle de resíduos (FLATEN et al., 2010).

O interesse em investigar o consumo de alimentos orgânicos na Amazônia vai além da preocupação com a saúde, implica em identificar se esta atividade pode ser inserida em um cenário futuro próximo como uma alternativa de produção sustentável tanto em relação a questão ambiental, quanto econômica para a região. Nesse contexto, o objetivo deste estudo foi caracterizar o mercado consumidor de alimentos orgânicos na cidade de Manaus-AM, bem como seus hábitos e principais motivações e limitações em relação ao consumo desses alimentos.

\section{MATERIAL E MÉTODOS}

A pesquisa foi desenvolvida na Feira do Produtor Orgânico (local regulamentado para a produção e comercialização de alimentos orgânicos, vinculada a Associação de Produtores Orgânicos do Estado do Amazonas - APOAM), localizada na sede do Ministério da Agricultura e Pecuária em Manaus-AM, que acontece todos os sábados de 07h30min às 11h30min. Foram investigados 100 usuários da feira, empregando um questionário que avaliou: os hábitos de consumo de alimentos orgânicos; os fatores que influenciam esse consumo e as características demográficas dos consumidores. As variáveis escolhidas foram agrupadas, segundo:

a) Perfil social do grupo consumidor: envolvendo as características relativas ao sexo, faixa etária, escolaridade e renda mensal;

b) Perfil de consumo: associado à definição (compreensão) de alimentos orgânicos; tipos de alimentos consumidos; motivos que levam ao consumo; quem consome nas residências (núcleo familiar); tempo de consumo; frequência de compra; como o consumidor identifica que o alimento adquirido é de origem orgânica e como o consumidor entende a relação destes com o meio ambiente. 
$\mathrm{Na}$ análise dos dados, a distribuição de frequências de agrupamentos de dados em classes na forma de frequência absoluta teve por finalidade reunir, segundo um critério de classificação, os valores em vários grupos, de tal forma que fosse possível avaliar a homogeneidade dentro do grupo e heterogeneidade entre grupos (ALBUQUERQUE et al., 2006).

A avaliação, segundo a aglomeração hierárquica, considerou o princípio das medidas de distância ou medidas de dissimilaridade (distância euclidiana). Nesta, se dois indivíduos são similares, eles estão próximos um do outro, ou seja, são comuns em relação ao conjunto de variáveis e viceversa (MAGALHÃES et al., 2008).

A pesquisa sobre preços foi realizada em três canais distintos de comercialização de alimentos orgânicos: a feira orgânica da APOAM e dois supermercados de grande porte da cidade. Nos locais pesquisados, os preços dos alimentos orgânicos foram comparados aos preços dos alimentos produzidos pelo cultivo convencional, presentes nos dois supermercados. A coleta de preços dos alimentos convencionais, nos supermercados, foi adotada por serem considerados canais modernos de comercialização de alimentos.

Nos supermercados não foram levados em consideração alimentos que passaram por processamento, somente os in natura foram selecionados. Os levantamentos foram realizados no intervalo de apenas 30 dias, com o objetivo de evitar a sazonalidade na produção, o que afetaria o resultado da pesquisa. Verificou-se que nem todos os alimentos orgânicos oferecidos na feira da APOAM estavam disponíveis nos supermercados. Sendo assim, foram selecionados para análise e comparação somente os produtos presentes em todos os canais de comercialização que fazem parte da pesquisa.

\section{RESULTADOS}

\section{Perfil do consumidor}

Dentre os consumidores pesquisados na Feira de Orgânicos da APOAM, foi detectado que $66 \%$ dos entrevistados são do sexo feminino e 34\% do masculino; $25 \%$ encontram-se na faixa etária de 30 a 40 anos, $21 \%$ entre 50 a 60 anos e $18 \%$ entre 40 a 50 anos; $34 \%$ possuem o ensino superior, $32 \%$ o ensino médio e $23 \%$ apresentam alguma forma de pós-graduação. Em termos de renda mensal destacase que $57 \%$ do público estão em uma faixa aproximadamente superior a 4,5 salários mínimos (SM); e 17\% concentram pessoas que declararam uma renda inferior a 1,5 SM (Figura 1). No referente ao conceito de alimento orgânico, $71 \%$ dos entrevistados afirmam ser um alimento sem agrotóxicos, livre de qualquer produto químico e $21 \%$ afirmam ser um produto que não agride o meio ambiente. Apenas $8 \%$ afirmaram ser um produto que não foi industrializado (Figura 2a). Os alimentos mais consumidos foram: as verduras, as frutas, os legumes e os ovos (Figura 2b). Os itens mais listados pelos consumidores foram: a alface, a rúcula, o agrião, a salsa, a couve, o mamão, a laranja, o tomate, o maracujá, o jambu, o cheiro verde, o limão, o abacaxi, a cenoura, o rabanete, a macaxeira, o maxixe, o feijão verde, os ovos, o jerimum e a banana.
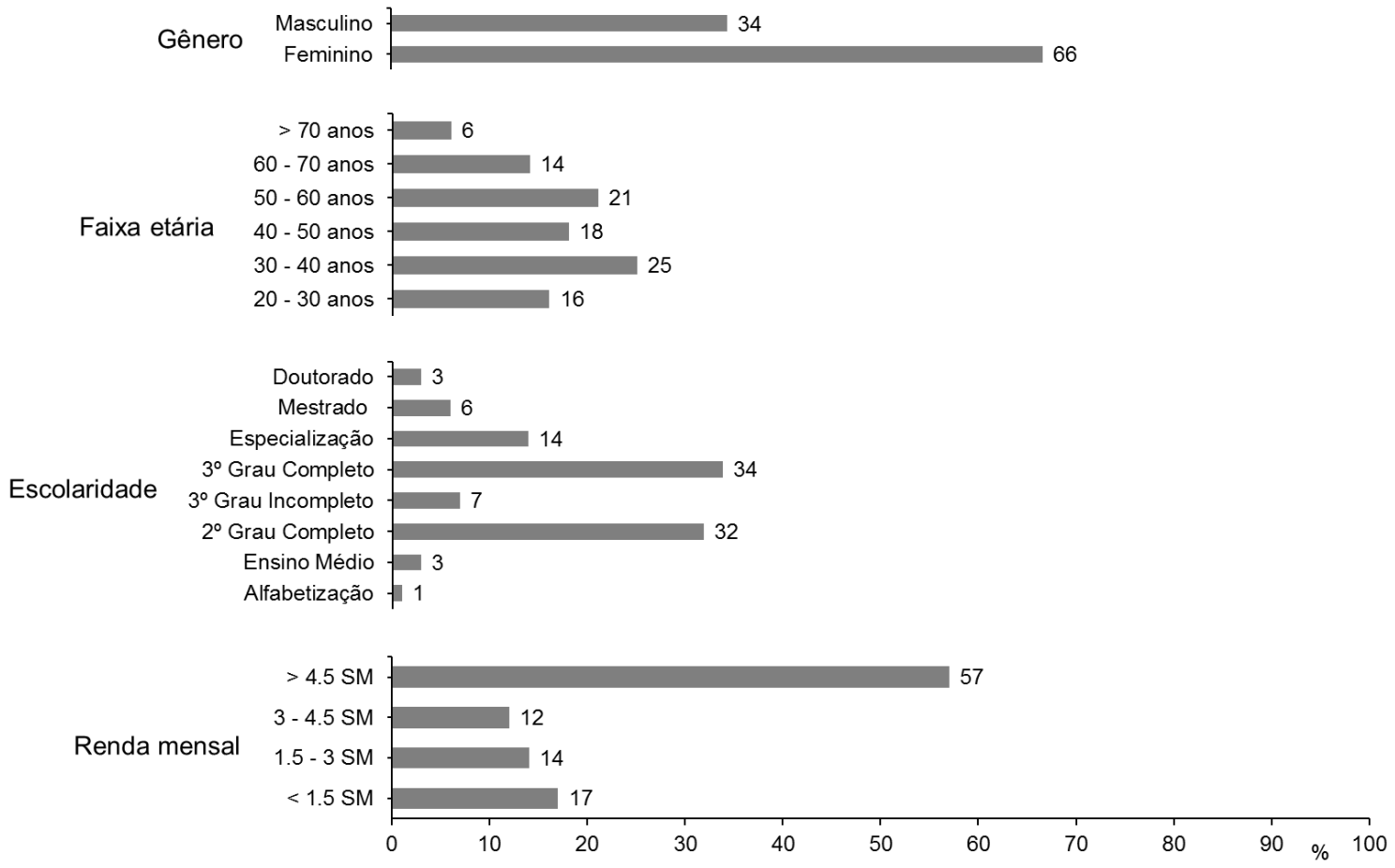

Figura 1. Perfil sociodemográfico dos consumidores de produtos orgânicos em Manaus-AM, Feira de Orgânicos da APOAM.

Os motivos que os levam a consumir alimentos orgânicos foram direcionados para a busca pela saúde e pela ingestão de alimento de qualidade e sem agrotóxicos (Figura 3a). Ao abordar sobre o consumo residencial de alimentos orgânicos, observou-se o predomínio da oferta de alimentos orgânicos para todos os membros da família (Figura 3b); sendo que $42 \%$ consomem orgânicos a aproximadamente 1 a 4 anos e $31 \%$ a mais de 5 anos (Figura 3c); com 84\% apresentando o hábito de frequentar a feira semanalmente (Figura 3d). 

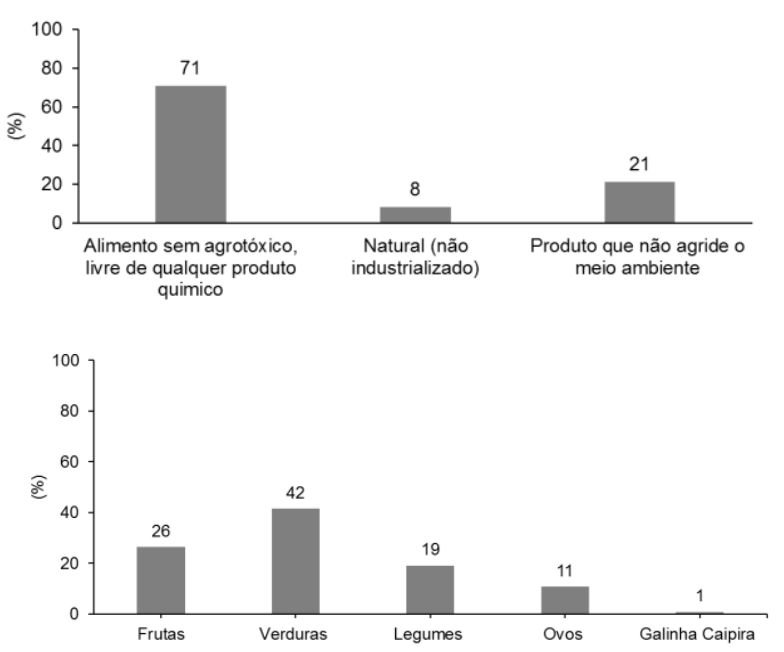

(b)

Figura 2. Frequência relativa: (a) conceito de alimento orgânico; (b) alimentos orgânicos consumidos.
A identificação dos produtos orgânicos é feita por $74 \%$ em função da palavra do produtor (Figura 4); porém, $96 \%$ acreditam que consumir este tipo de alimento está contribuindo para a preservação ambiental, e 4\% declaram-se indiferentes quanto a isto.

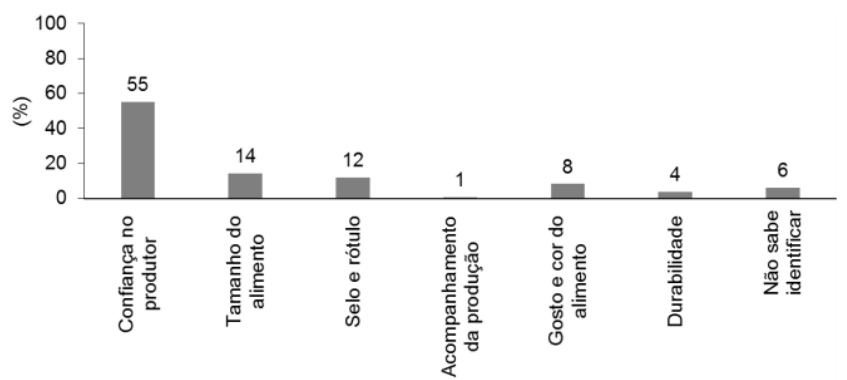

Figura 4. Frequência relativa quanto a forma de identificação se um alimento é orgânico ou não.
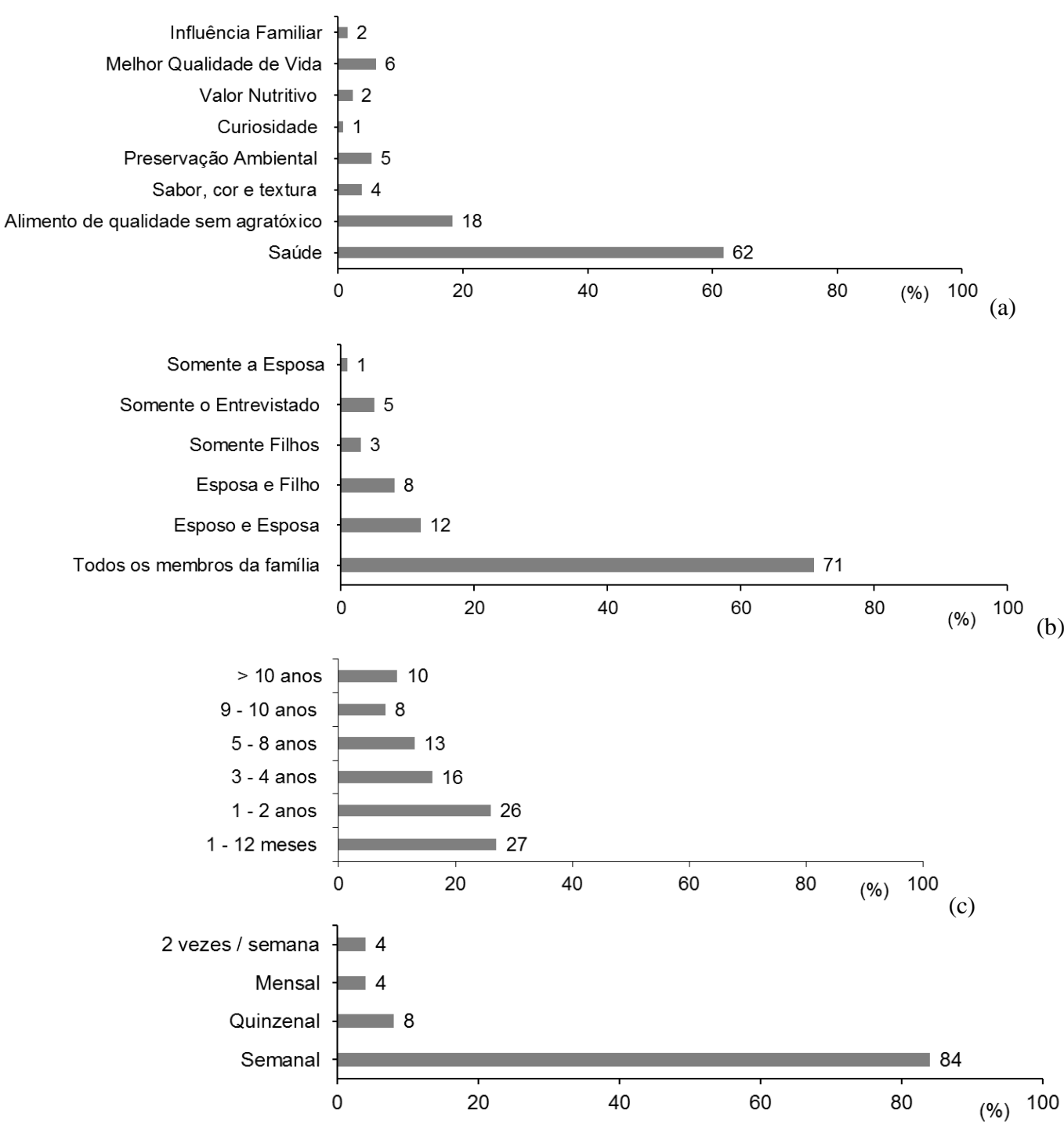

(d)

Figura 3. Frequência relativa: (a) motivação de consumo; (b) alimentos orgânicos consumidos; (c) tempo de consumo; (d) Frequência de visitação a feira. 


\section{Indicadores do perfil consumidor}

$\mathrm{Na}$ aplicação das distribuições estatísticas, observa-se que alguns parâmetros avaliados condicionam o perfil do consumidor, permitindo uma comparação entre os fatores conjugados. A análise por agrupamentos (Figura 5) demonstra uma associação entre: a "Faixa etária" e o "Tempo de consumo"; o "Consumo residencial", a "Identificação do alimento" e a "Definição de alimento orgânico"; e a "Frequência de compra" e o "Motivo do consumo". As variáveis de "Escolaridade" e "Renda mensal" mostraram um comportamento mais transversal, porém vinculados ao ramo que define a "Faixa etária" e o "Tempo de consumo".

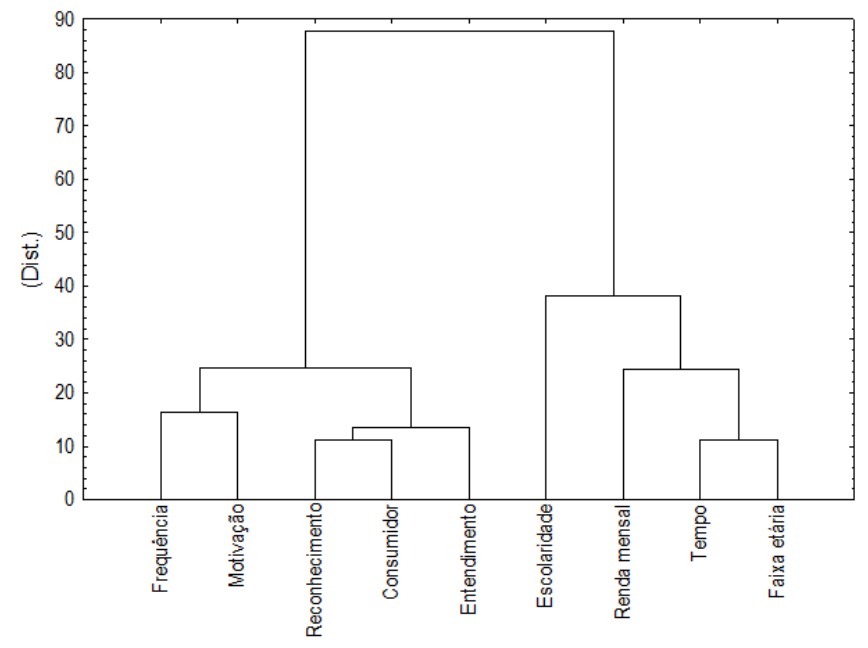

Figura 5. Análise de agrupamentos: Frequência - Frequência de compra; Motivação - Motivo do consumo; Reconhecimento - Identificação do alimento; Consumidor Consumo residencial; Entendimento - Definição de alimento orgânico; Escolaridade; Renda mensal; Tempo - Tempo de consumo; e Faixa etária.

Como a comercialização de alimentos orgânicos na feira iniciou em 2009, as pessoas que consomem tais produtos há quatro anos, se tornaram um público fiel e constante. Este, é em geral maduro (30-60 anos) e com uma renda mensal que caracteriza um perfil de profissionais graduados. Portilho et al. (2011) destacam um relativo crescimento da liberdade de escolha dos consumidores conectado a uma busca pela emancipação, identidade pessoal e autonomia, que somente seriam alcançados com uma sociedade mais esclarecida e com maior liberdade sobre o que decidir e escolher.

O reconhecimento do que é um produto orgânico no ato da compra ainda é deficiente, com uma baixa $(<10 \%)$ preocupação em avaliar rótulos e buscar certificações no momento da aquisição dos alimentos (PORTILHO, 2010); o que não impede do produto ser ofertado para toda a família, uma vez que acredita-se que ele seja um produto saudável pela sua desassociação ao uso de agrotóxicos. Archanjo et al. (2001) e Portilho e Castaneda (2011) argumentam que o contato direto do consumidor com o produtor cria uma relação de confiança com o produto orgânico, cuja procedência se atesta independentemente da presença do selo ou do certificado.

A frequência semanal dos consumidores mostrou-se relacionada ao principal fator motivador que foi o cuidado com a saúde. Em Hoefkens et al. (2009) e Lairon (2010) observa-se que quando uma pessoa é motivada a comprar um alimento orgânico, existe uma disposição em adquirir um produto sem aditivos químicos e produzidos de acordo com as normas de produção orgânicas. Sousa et al. (2012) alegam que existe o argumento de que uma dieta orgânica pode diminuir a exposição a pesticidas, porém existe uma margem de erro quando se comparam pessoas que consomem habitualmente alimentos orgânicos com aquelas que consomem alimentos convencionais com grande número de variáveis não controladas.

De forma geral, o perfil do consumo de produtos orgânicos, verificado em Manaus (Tabela 1) é semelhante aos registrados em trabalhos efetuados em Goiânia (GO), Porto Alegre (RS), Belo Horizonte (MG), Rio de Janeiro (RJ) e Curitiba (PR); principalmente nas variáveis definidoras do perfil do grupo social (faixa etária, escolaridade, renda mensal e sexo). Para Ferraz et al. (2013) essa segmentação, segundo um perfil específico para o consumo de orgânicos, é decorrente de diversos fatores dentre estes citam-se: o preço de venda desses produtos; a baixa diversidade; a falta de confiança na procedência do alimento; o menor tempo de vida útil e a baixa regularidade de fornecimento.

Existindo uma abordagem uniforme, conforme Portilho (2010) e Silva et al. (2013), quanto ao fato destes consumidores enxergarem no ato de adquirir produtos orgânicos não somente a o valor nutritivo, mas também o fortalecimento da agricultura baseada em práticas sustentáveis.

\section{Preços ao Consumidor dos Alimentos Orgânicos em Manaus - AM}

A Tabela 2 apresenta o resultado da pesquisa sobre preços praticados no mercado, tendo sido selecionados os seguintes alimentos orgânicos e convencionais para a análise: tomate, hortelã, jerimum, couve, limão, mamão, coentro, cebolinha, salsa, chicória, ovo, gengibre, rúcula, alface americana, brócolis, banana, cenoura, tomate-cereja, gengibre, pimenta-de-cheiro, pepino, macaxeira, inhame e laranja.

Ressalta-se a dificuldade de relacionar os preços obtidos na feira com os preços dos supermercados, pois nestes os preços são em quilograma e na feira, em razão de os produtores exercerem uma administração muito empírica, os preços são estipulados em unidades, maços e sacolas de diversos tamanhos. Por isso, acredita-se que o consumo de alimentos orgânicos liga-se mais aos benefícios percebidos pelos consumidores quando a ingestão destes alimentos do que com o valor cobrado pelos vendedores destes produtos.

Apesar das diferenças quanto às unidades, observa-se que pelo tamanho dos maços e sacolas ofertados pelos produtores na feira de orgânicos o alimento orgânico ainda é mais caro que o alimento produzido de forma convencional, quando comparamos visualmente com os ofertados nos supermercados, resultado semelhante foi obtido por Barbosa et al. (2011) na análise do comércio de produtos orgânicos em Goiânia. 
Tabela 1 - Comparativo do perfil do consumo em Manaus com outras capitais brasileiras.

\begin{tabular}{|c|c|c|c|c|c|c|}
\hline \multirow[b]{2}{*}{ Variáveis } & \multicolumn{6}{|c|}{ Perfil dominante } \\
\hline & Manaus (AM) & Goiânia $(\mathrm{GO})^{1}$ & $\begin{array}{l}\text { Porto Alegre } \\
(\mathrm{RS})^{2}\end{array}$ & $\begin{array}{l}\text { Belo Horizonte } \\
(\mathrm{MG})^{3}\end{array}$ & $\begin{array}{c}\text { Rio de Janeiro } \\
(\mathrm{RJ})^{4}\end{array}$ & $\begin{array}{l}\text { Curitiba } \\
(\mathrm{PR})^{5}\end{array}$ \\
\hline Sexo & Feminino & Feminino & Feminino & Feminino & Feminino & Feminino \\
\hline Escolaridade & $\begin{array}{l}3^{\circ} \text { Grau } \\
\text { completo }\end{array}$ & $\begin{array}{l}3^{\circ} \mathrm{Grau} \\
\text { completo }\end{array}$ & $\begin{array}{l}2^{\circ} \mathrm{Grau} \\
\text { completo }\end{array}$ & $\begin{array}{l}3^{\circ} \text { Grau } \\
\text { completo }\end{array}$ & $\begin{array}{l}3^{\circ} \mathrm{Grau} \\
\text { completo }\end{array}$ & $\begin{array}{c}3^{\circ} \text { Grau } \\
\text { completo }\end{array}$ \\
\hline Idade & $30-70$ anos & $\begin{array}{l}\text { Maior que } 30 \\
\text { anos }\end{array}$ & $\begin{array}{l}\text { Maior que } 30 \\
\text { anos }\end{array}$ & $\begin{array}{l}\text { Maior que } 30 \\
\text { anos }\end{array}$ & $\begin{array}{l}\text { Maior que } 30 \\
\text { anos }\end{array}$ & $\begin{array}{l}\text { Maior que } 30 \\
\text { anos }\end{array}$ \\
\hline Renda & $\begin{array}{l}\text { Mais que } 4,5 \\
\text { salários } \\
\text { mínimos } \\
\end{array}$ & $\begin{array}{l}3 \text { a } 4 \text { salários } \\
\text { mínimos }\end{array}$ & $\begin{array}{l}\text { Maior que } 3 \\
\text { salários } \\
\text { mínimos } \\
\end{array}$ & $\begin{array}{l}\text { Maior que } 9 \\
\text { salários } \\
\text { mínimos } \\
\end{array}$ & $\begin{array}{l}\text { Maior que } 7 \\
\text { salários mínimos }\end{array}$ & $\begin{array}{l}\text { Maior que } 12 \\
\text { salários } \\
\text { mínimos }\end{array}$ \\
\hline Motivação & Saúde & Saúde & $\begin{array}{l}\text { Saúde e } \\
\text { preocupação } \\
\text { ambiental }\end{array}$ & Saúde & $\begin{array}{l}\text { Saúde e } \\
\text { preocupação } \\
\text { ambiental }\end{array}$ & Saúde \\
\hline $\begin{array}{l}\text { Frequência da } \\
\text { compra }\end{array}$ & Semanal & Semanal & Semanal & Semanal & Semanal & Semanal \\
\hline $\begin{array}{l}\text { Identificação } \\
\text { do alimento }\end{array}$ & $\begin{array}{l}\text { Confiança no } \\
\text { produtor }\end{array}$ & $\begin{array}{l}\text { Origem do } \\
\text { produto e } \\
\text { credibilidade } \\
\text { do vendedor }\end{array}$ & $\begin{array}{l}\text { Confiança no } \\
\text { produtor }\end{array}$ & $\begin{array}{l}\text { Local de } \\
\text { compra }\end{array}$ & & $\begin{array}{l}\text { Confiança no } \\
\text { produtor }\end{array}$ \\
\hline
\end{tabular}

${ }^{1}$ Barbosa et al. (2011); ${ }^{2}$ Hope et al. (2013); ${ }^{3}$ Andrade e Bertoldi (2012); ${ }^{4}$ Silva et al. (2013); ${ }^{5}$ Rucinski e Brandenburg (2009).

Tabela 2. Comparação dos preços orgânicos x convencional.

\begin{tabular}{|c|c|c|c|}
\hline Produto & Supermercado - A & Supermercado - B & Feira de Orgânicos \\
\hline Tomate & $\mathrm{R} \$ 7,82(\mathrm{~kg})$ & $\mathrm{R} \$ 7,79(\mathrm{~kg})$ & $\mathrm{R} \$ 4,00$ (sacola) \\
\hline Tomate-cereja & $\mathrm{R} \$ 22,75(\mathrm{~kg})$ & $\mathrm{R} \$ 29,66(\mathrm{~kg})$ & $\mathrm{R} \$ 14,28(\mathrm{~kg})$ \\
\hline Jerimum & $\mathrm{R} \$ 2,78(\mathrm{~kg})$ & $\mathrm{R} \$ 2,79(\mathrm{~kg})$ & $\mathrm{R} \$ 3,00(\mathrm{~kg})$ \\
\hline Couve & $\mathrm{R} \$ 1,49$ (maço) & $\mathrm{R} \$ 1,50$ (maço) & $\mathrm{R} \$ 2,00$ (maço) \\
\hline Limão & $\mathrm{R} \$ 3,25(\mathrm{~kg})$ & $\mathrm{R} \$ 3,25(\mathrm{~kg})$ & $\mathrm{R} \$ 3,00$ (maço) \\
\hline Mamão & $\mathrm{R} \$ 5,85(\mathrm{~kg})$ & $\mathrm{R} \$ 7,95(\mathrm{~kg})$ & $\mathrm{R} \$ 5,00(\mathrm{~kg})$ \\
\hline Coentro & $\mathrm{R} \$ 2,19$ (maço) & $\mathrm{R} \$ 2,85$ (maço) & $\mathrm{R} \$ 2,50$ (maço) \\
\hline Cebolinha & $\mathrm{R} \$ 2,22$ (maço) & $\mathrm{R} \$ 2,65$ (maço) & $\mathrm{R} \$ 2,50$ (maço) \\
\hline Chicória & $\mathrm{R} \$ 2,19$ (maço) & $\mathrm{R} \$ 2,85$ (maço) & $\mathrm{R} \$ 1,00$ (maço) \\
\hline Salsa & $\mathrm{R} \$ 4,10$ (maço) & $\mathrm{R} \$ 4,29$ (maço) & $\mathrm{R} \$ 2,50$ (maço) \\
\hline Gengibre & $\mathrm{R} \$ 23,68(\mathrm{~kg})$ & $\mathrm{R} \$ 24,10(\mathrm{Kg})$ & $\mathrm{R} \$ 15,00(\mathrm{~kg})$ \\
\hline Rúcula & $\mathrm{R} \$ 2,42$ (maço) & $\mathrm{R} \$ 3,70$ (maço) & $\mathrm{R} \$ 2,50$ (maço) \\
\hline Alface Americana & $\mathrm{R} \$ 4,71$ (maço) & $\mathrm{R} \$ 4,59$ (maço) & $\mathrm{R} \$ 4,00$ (maço) \\
\hline Brócolis & $\mathrm{R} \$ 43,01(\mathrm{~kg})$ & $\mathrm{R} \$ 38,54(\mathrm{Kg})$ & $\mathrm{R} \$ 5,00$ (maço) \\
\hline Macaxeira & $\mathrm{R} \$ 2,59(\mathrm{~kg})$ & $\mathrm{R} \$ 7,69(\mathrm{~kg})$ & $\mathrm{R} \$ 5,00(\mathrm{~kg})$ \\
\hline Laranja & $\mathrm{R} \$ 3,02(\mathrm{~kg})$ & $\mathrm{R} \$ 12,90(\mathrm{~kg})$ & $\mathrm{R} \$ 5,00$ (dúzia) \\
\hline Ovos & $\mathrm{R} \$ 14,35$ (cartela) & $\mathrm{R} \$ 14,39$ (cartela) & $\mathrm{R} \$ 15,00$ (cartela) \\
\hline Banana Prata & $\mathrm{R} \$ 5,54(\mathrm{~kg})$ & $\mathrm{R} \$ 4,89(\mathrm{~kg})$ & $\mathrm{R} \$ 5,00$ (palma) \\
\hline Maxixe & $\mathrm{R} \$ 18,30(\mathrm{~kg})$ & $\mathrm{R} \$ 29,67(\mathrm{~kg})$ & $\mathrm{R} \$ 2,50$ (sacola) \\
\hline Feijão-verde & $\mathrm{R} \$ 2,50$ (maço) & $\mathrm{R} \$ 3,10$ (maço) & $\mathrm{R} \$ 2,50$ (maço) \\
\hline Pepino & $\mathrm{R} \$ 2,80(\mathrm{Kg})$ & $\mathrm{R} \$ 2,98(\mathrm{Kg})$ & $\mathrm{R} \$ 5,00$ (pacote) \\
\hline Pimenta-de-Cheiro & $\mathrm{R} \$ 15,70(\mathrm{Kg})$ & $\mathrm{R} \$ 15,28(\mathrm{Kg})$ & $\mathrm{R} \$ 10,00(\mathrm{~kg})$ \\
\hline Inhame & $\mathrm{R} \$ 8,90(\mathrm{Kg})$ & $\mathrm{R} \$ 7,69(\mathrm{Kg})$ & $\mathrm{R} \$ 10,00(\mathrm{~kg})$ \\
\hline Hortelã & $\mathrm{R} \$ 5,00$ (maço) & $\mathrm{R} \$ 5,80$ (maço) & $\mathrm{R} \$ 3,00$ (maço) \\
\hline
\end{tabular}

Os produtores da APOAM relataram outras questões que influenciam no preço dos produtos, tais como: as dificuldades logísticas de transporte até Manaus, a variabilidade climática que influencia no plantio na região e a falta de investimentos públicos no fomento a instrumentalização e capacitação do produtor.

Para Momesso et al. (2009), na cadeia de alimentos orgânicos, o consumidor final é o elemento principal; ou seja, não havendo demanda, não há aumento de produção, não há investimentos por parte do poder público, não há interesse em melhorar a tecnologia e assim por diante. A partir do exposto em Barbosa et al. (2011) e Portilho e Castaneda (2011) observa-se que existe uma tendência para que a diferença percentual entre produtos orgânicos e convencionais decresça, porém existe a dependência das taxas de crescimento da demanda e da oferta, além do fortalecimento de formas de certificação que garantam a procedência e a qualidade do alimento. 


\section{CONCLUSÕES}

O crescimento do consumo de alimentos orgânicos está diretamente relacionado com os diversos significados que este alimento traz para os seus consumidores e a internalização de uma cultura que busca a sustentabilidade ambiental e o resgate de valores. A Feira do Produtor Orgânico, em Manaus, busca atender os consumidores que procuram alimentos orgânicos, promovendo um mercado produtor cujo crescimento vem aumentando desde 2009, ano em que iniciou-se a feira.

Estes consumidores tem um perfil concordante com outras cidades do país, marcado por um público disposto a pagar pelo alimento, que apesar de demonstrar escolaridade, ainda realiza a compra de forma empírica, confiando no produtor. São clientes e consumidores fiéis do consumo de produtos orgânicos, considerando que semanalmente visitam a feira, para a compra de verduras, frutas e legumes, os quais são oferecidos para todos os membros da família.

A identificação do perfil dos consumidores do alimento orgânico e a sua representatividade social contribuem com a orientação da cadeia produtiva, desde a produção até o desenvolvimento de estratégias de marketing que podem potencializar o consumo deste tipo de produto alimentício. Tais estratégias são necessárias, visto que detectou-se que há pouca oferta de variedade e quantidade dos alimentos e o valor dos produtos vendidos são entraves encontrados pelos produtores.

\section{REFERÊNCIAS BIBLIOGRÁFICAS}

ALBUQUERQUE, M. A. F.; FERREIRA, R. L. C.; SILVA, J. A. A.; Santos, E. S.; STOSIC, B.; SOUZA, A. L. Estabilidade em análise de agrupamento: estudo de caso em ciência florestal. Revista Árvore, v. 30, n. 2, p. 257 265, 2006.

ALBUQUERQUE, A. A arte de não adoecer. Manaus: Fama, 2011, 128p.

ANDRADE, L. M. S.; BERTOLDI, M. C. Atitudes e motivações em relação ao consumo de alimentos orgânicos em Belo Horizonte - MG. Brazilian Journal of Food Technology, p. 31-40, 2012.

ARCHANJO, L. R.; BRITO, K. F. W.; SAUERBECK, S. Alimentos Orgânicos em Curitiba: consumo e significado. Rev. Caderno de Debates,v. 8, p. 1-6, 2001.

BARBOSA, S. C; MATTEUCCI, M. B. A.; LEANDRO, W. M.; LEITE, A. F.; CAVALCANTE, E. L. S.; ALMEIDA, G. Q. E. Perfil do consumidor e oscilações de preços de produtos agroecológicos. Pesquisa Agropecuária Tropical, v. 41, p. 602-609, 2011.

BORGUINI, R. G.; TORRES, E. A. F. S. Alimentos Orgânicos: qualidade nutritiva e segurança do alimento. Rev. Segurança Alimentar e Nutricional, v. 13, p. 6475, 2006.

BOURN, D.; PRESCOTT, J. A comparison of the nutritional value, sensory qualities, and food safety of organically and conventionally produced foods. Crit. Rev. Food Sci. Nutr., v. 42, p. 1-34, 2002.

CREMONEZI, G.; PIZZINATTO, N.; SPERS, V. Estratégia e Desenvolvimento de Produtos no Agronegócio: um estudo em produtos orgânicos. Rev. Adm. MADE. v. 15, n. 3, p. 85-89. 2011.

DIAZ, F. J. M.; PLEITE, F. M. C.; MARTINEZ-PAZ, J. M.; GARCIA, P. G. Willingness to pay for organic food in Spain: an approach to the analysis of regional differences. Itea-Informacion Tecnica Economica Agraria, v. 107, n. 1, p. 3-20, 2011.

FLATEN, O.; LIEN, G.; KOESLING, M.; LOES, A. K. Norwegian farmers ceasing certified organic production: characteristics and reasons. J. Environ. Manag., v. 91, n. 12, p. 2717-2726, 2010.

FERRAZ, A. P. C. R.; MALHEIROS, J. M.; CINTRA, R. M. G. A produção, o consumo e a composição química dos alimentos orgânicos. Revista Simbio-Logias, v. 6, n. 9, p. 32-42, 2013.

HOEFKENS, C.; VERBEKE, W.; AERTSENS, MONDELAERS K.; VAN CAMP J. The nutritional and toxicological value of organic vegetables: Consumer perception versus scientific evidence. British Food Journal, v. 111, n. 10, p. 1062-1077, 2009.

HOPPE, Al.; VIEIRA, L. M.; BARCELLOS, M. D. Consumer behaviour towards organic food in Porto Alegre: an application of the theory of planned behaviour. Rev. Econ. Sociol. Rural, v. 51, n. 1, p. 69-90, 2013.

LACAZE, V. Consumos alimentarios sustentables en Argentina: una estimacion de la disposicion a pagar por alimentos organicos frescos y procesados porconsumidores de la ciudad de Buenos Aires. Revista Agroalimentaria. v. 15, n. 29, p. 87-100, 2009.

LAIRON, D. Nutritional quality and safety of organic food: a review. Agron. Sustain. Dev., v. 30, p. 33-41, 2010.

LIMA, G. P. P.; VIANELO, F. Review on main differences between organic and conventional plant based foods. Int. J. Food Sci. Technol., v. 46, p. 1-11, 2011.

MAGALHÃES, L. P.; OSHIMA, C. T. F.; SOUZA, L. G.; LIMA, J. M.; CARVALHO, L.; FORONES, N. M. Variação de peso, grau de escolaridade, saneamento básico, etilismo, tabagismo e hábito alimentar pregresso em pacientes com câncer de estômago. Arquivos de Gastroenterologia, v. 45, n. 2, p. 111-116, 2008.

MOMESSO, C. M. V.; ROEL, A. R.; FAVARO, S. P. Levantamento do potencial de comercialização de produtos orgânicos para o Estado de Mato Grosso do Sul. Interações, v. 10, n. 1, p. 55-62, 2009.

NESS, M. R.; NESS, M.; BRENNAN, M.; OUGHTON, E.; RITSON, C.; RUTO, E. Modelling consumer behavioural 
intentions towards food with implications for marketing quality low-input and organic food. Food Quality and Preference, v. 21, n. 1, p. 100-111, 2010.

PORTILHO, F. Autoatribuição de responsabilidade: consumo de alimentos orgânicos em uma feira certificada. Etnográfica, v. 14, n. 3, p. 549-565, 2010.

PORTILHO, F.; CASTANEDA, M. Certificação e confiança face a face em feiras de produtos orgânicos. Revista de Economia Agrícola, v. 58, n. 1, p. 11-21, 2011.

PORTILHO, F., CASTAÑEDA, M; CASTRO, I. R. R. A Alimentação no contexto contemporâneo: consumo, ação política e sustentabilidade. Revista Ciência e Saúde Coletiva, v. 16. n. 1, p. 99-106, 2011.

ROITNER-SCHOBESBERGER， B.; DARNHOFER， I.; SOMSOOK, S.; VOGL, C. R. Consumer perceptions of organic foods in Bangkok, Thailand. Food Policy, v. 33, n. 1, p. 112-121, 2008.

RUCINSKI, J.; BRANDENBURG, A. Consumidores de alimentos orgânicos em Curitiba. In: ENCONTRO DA ANPPAS, 1, Indaiatuba. Anais... Indaiatuba: ANPPAS, 2002.
SANTOS, G. C.; MONTEIRO, M. Sistema Orgânico de Produção de Alimentos. Alimentos e Nutrição, v. 15, n. 1, p. 73-86, 2004.

SILVA, E. B.; CARDOSO, F. T.; SOUZA.G. G.; ALMEID, A. Perfil sócio econômicos de consumidores de produtos orgânicos. Revista Verde, v. 8, n. 1, p. 83 - 89, 2013.

SOUSA, A. A.; AZEVEDO, E.; LIMA, E. E.; SILVA, A. P. F. Alimentos orgânicos e saúde humana: estudo sobre as controvérsias. Rev Panam Salud Publica, v. 31, n. 6, p. 513-7, 2012.

TERRAZZA, P.; VALARINI, P. J. Situação do mercado de Produtos Orgânicos e as Formas de Comercialização no Brasil. Rev. Informações Econômicas, v. 39, n. 11, p. 27-41, 2009.

YIRIDOE, E. K.; BONTI-ANKOMAH, S. MARTIN, R. C. Comparison of consumer perception and preference toward organic versus conventionally produced foods: A review and update of the literature. Renew. Agric. Food. Syst., v. 20, p. 193-205, 2005. 